The Astrophysical Journal, 223:824-834, 1978 August 1

(C) 1978. The American Astronomical Society. All rights reserved. Printed in U.S.A.

\title{
CALIBRATION OF METALLICITY EFFECTS ON THE INTEGRATED COLORS OF GLOBULAR CLUSTERS AND EARLY-TYPE GALAXIES
}

\author{
MARC AARONSON* \\ Steward Observatory, University of Arizona \\ Judith G. COHEN AND JeRemy Mould \\ Kitt Peak National Observatory $\dagger$ \\ AND \\ Matthew MalKan* \\ Center for Astrophysics, Harvard College Observatory and Smithsonian Astrophysical Observatory \\ Received 1977 December 9; accepted 1978 February 8
}

\begin{abstract}
New infrared observations of globular clusters have been obtained which show that both infrared and optical colors are strongly correlated with metallicity and which provide an empirical calibration of abundance effects in composite stellar systems. Models have been constructed, based on the isochrones of Ciardullo and Demarque, with $Z$-values between 0.0001 and 0.04 , and slope of the initial mass function $s$ between 0 and 4. Metal-poor models with $s \leq 2.35$ (the Salpeter function) give good agreement with the empirical calibration. Metal-rich models are compared with observations of the central regions of early-type galaxies, and imply that galaxies which have $-19 \geq M_{V} \geq-23$ correspond to a range in metallicity of $0.0 \leq[\mathrm{M} / \mathrm{H}] \leq+0.3$. Models with $s=2.35$ adequately fit the observations; proper accounting of metallicity effects on narrow-band infrared features does not require $s<2$, as previously published models have suggested. An upper limit on $s$ of 3.2 is determined.
\end{abstract}

Subject headings: cluster: globular - galaxies: stellar content — stars: abundances

\section{INTRODUCTION}

In recent years a large effort has been devoted to analyzing the integrated light of early-type galaxies (see, e.g., Tinsley 1972; Faber 1972; O'Connell 1976; Tinsley and Gunn 1976a; Whitford 1977). Because both observational data and proper theoretical tools have heretofore been lacking, published models have almost always assumed calibrations based on solar abundances. However, it has often been argued that the well-known color-luminosity and color-aperture relations in early-type galaxies are at least partly due to metallicity variations (McClure and van den Bergh 1968; Sandage 1972; Strom et al. 1976, 1978; Faber 1977; Frogel et al. 1978, hereafter Paper I). In particular, Faber (1973) concluded, from a detailed study of optical line indices, that the central regions of elliptical galaxies could be characterized by a oneparameter family in absolute magnitude, with the brightest galaxies being metal-rich.

In this paper we first present new infrared observations of globular clusters which, in combination with broad-band optical colors, provide a strictly empirical calibration of integrated color change with metallicity. The integrated light of globular clusters should become redder with increased metallicity because of (1) an

* Guest investigator at Kitt Peak National Observatory.

$\dagger$ Operated by the Association of Universities for Research in Astronomy, Inc., under contract with the National Science Foundation. increase in line blanketing, (2) a shift of the horizontal branch from the blue to the red, and (3) cooling of the giant branch. Unfortunately, line blanketing effects are notoriously difficult to assess, and the position of the horizontal branch is known to be imperfectly correlated with metallicity (cf. Sandage and Wildey 1967). However, infrared colors are primarily affected by the position of the giant branch and thus, in theory, should provide a more straightforward measurement of metallicity than broad-band optical colors. We shall see that this is, in fact, the case.

In order to provide a theoretical fit to the globular cluster data, models are constructed with varying metallicity $[\mathrm{M} / \mathrm{H}]$ and initial mass function (IMF) from the theoretical isochrones of Ciardullo and Demarque (1977, hereafter CD). Separation of the effects of $[\mathrm{M} / \mathrm{H}]$ variation from a change in $s,{ }^{1}$ the slope of the IMF, is an especially crucial problem for galaxies because both the stellar mass-to-light ratio $(M / L)$ and the evolutionary color corrections depend strongly on $s$ (Tinsley 1972).

Finally, the model results are compared with observations of early-type galaxies in order to determine the most likely values of $s$ and $[\mathrm{M} / \mathrm{H}]$ in such galaxies. Since the models accurately reproduce the empirical color-metallicity relation, we can have some confidence in the validity of this comparison.

\footnotetext{
${ }^{1}$ The quantity $s=1+x$, where $x$ is the slope of the IMF in the notation of Tinsley (1972).
} 
The observations and empirical calibration are presented in $\S$ II, the model construction is discussed in $\S$ III, and the results are analyzed in $\S$ IV. Our conclusions are summarized in $\S \mathrm{V}$.

\section{OBSERVATIONS AND EMPIRICAL CALIBRATION}

Our empirical calibration is based on broad-band $J K$ measurements of the central regions of 42 globular clusters combined with previously published UV observations. In addition, narrow-band measurements of the $2.4 \mu \mathrm{m} \mathrm{CO}$ and $1.9 \mu \mathrm{m} \mathrm{H}_{2} \mathrm{O}$ absorption features were obtained for about two-thirds of the sample. These latter features are sensitive measures of luminosity and effective temperature in late-type stars (Baldwin, Frogel, and Persson 1973; Paper I; Aaronson, Frogel, and Persson 1978, hereafter Paper II).

Infrared data for five of the clusters were taken from Papers I and II. The remaining 37 clusters were measured in the infrared during 1976 and 1977 on the Kitt Peak No. $10.9 \mathrm{~m}$ telescope with the Harvard College Observatory InSb detector system. A focalplane chopper and offset guider were used for all measurements.

The statistical accuracy of the infrared data was always $\lesssim 0.02$ mag for all filters. Repetition of about one-third of the measurements on different nights of the same observing run, and also on different observing runs, showed that the photometric errors were comparable with the statistical errors. The infrared beam sizes for the cluster data in this paper were $105^{\prime \prime}$ for 39 clusters, $84^{\prime \prime}$ for two clusters, and 66" for one cluster. The ratio of beam chopper throw to beam diameter was 1.5 to 2.5 , so that the correction for flux in the "reference" beam was quite small. For 39 clusters, this correction was $\leq 0.05 \mathrm{mag}$, while for the remaining three clusters the correction ranged between 0.1 and $0.2 \mathrm{mag}$. The data were also corrected for beam profile effects following the procedures in Paper I. The photometric system (which, for $J$ and $K$, is essentially the same as that of Johnson 1966) is discussed fully in Paper II and in Aaronson (1978). A full presentation of the infrared data, including multiaperture measurements, will be given in Aaronson, Malkan, and Kleinmann (1978).
$V-K$ colors were formed for 38 globular clusters by using the magnitudes published by Kron and Mayall (1960), transformed to the $V$ system as in Peterson and King (1975). We believe the uncertainty in the $V-K$ colors introduced by this transformation is offset by the fact that all of the Kron and Mayall (1960) measurements are on a uniform system. Integrated $U-V$ colors were adopted from the compilation of Harris and van den Bergh (1974).

The data have been corrected for reddening by using color excess values of $E(B-V)$ from Kron and Guetter (1976) and the Whitford reddening curve (Johnson 1968). For five clusters not measured by Kron and Guetter (1976), the mean of the reddening values listed by Harris (1976) and by Burstein and McDonald (1975) were adopted.

The empirical metallicity calibration was derived from only those clusters with metallicity determinations based on abundance measurements in individual stars. The calibrating clusters, adopted values of $[\mathrm{M} / \mathrm{H}]$, and sources of these values are summarized in Table 1 . For the $[M / H]$ values taken from Hesser, Hartwick, and McClure's (1977) Table 2, $[\mathrm{M} / \mathrm{H}]=$ -0.3 was adopted for NGC 6838 , and the remaining cluster values were adjusted according to their interpolation scheme. For the clusters not listed in Table 1, values of $[M / H]$ given in Kukarkin (1975) were assumed.

In Figure 1 various colors are plotted against $[\mathrm{M} / \mathrm{H}]$. We have adopted nominal errors of $0.08,0.15$, $0.04,0.025$, and $0.025 \mathrm{mag}$, and $0.2 \mathrm{dex}$ for $U-V$, $V-K, J-K, \mathrm{CO}, \mathrm{H}_{2} \mathrm{O}$, and $[\mathrm{M} / \mathrm{H}]$, respectively. The last applies to the calibrating clusters onlyvalues of $[\mathrm{M} / \mathrm{H}]$ from Kukarkin (1975) are probably no better than $\pm 0.4 \mathrm{dex}$. The large error adopted for $V-K$ reflects uncertainties in (1) the transformation of the Kron and Mayall (1960) magnitudes to the $V$ system, (2) the reference beam corrections to the $K$ magnitudes, and (3) matching of the $V$ and $K$ aperture sizes. (Because globular clusters have steep growth curves [Kron and Mayall 1960; King 1966] at the typical infrared beam size used here, an error of $3^{\prime \prime}$ in matching the $V$ and $K$ aperture can lead to a 0.05 mag shift in the $V-K$ color.)

Least-squares fits to the data were made by using the calibrators only and are shown in Figure 1. To

TABLE 1

Metallicity Calibrators

\begin{tabular}{ccl|ccc}
\hline \hline NGC & $\begin{array}{c}\text { Adopted } \\
{[\mathrm{M} / \mathrm{H}]}\end{array}$ & \multicolumn{1}{c|}{ Sources* } & NGC & $\begin{array}{c}\text { Adopted } \\
{[\mathrm{M} / \mathrm{H}]}\end{array}$ & Sources* \\
\hline $5024 \ldots \ldots \ldots$ & -1.7 & 1,2 & $6341 \ldots \ldots \ldots$ & -2.2 & $1,2,3,4$ \\
$5272 \ldots \ldots$ & -1.6 & $1,2,3,4$ & $6656 \ldots \ldots \ldots$ & -1.8 & 1,2 \\
$5904 \ldots \ldots$ & -1.1 & 1,3 & $6838 \ldots \ldots$ & -0.3 & $1,2,3,5$ \\
$6121 \ldots \ldots$ & -1.2 & 1 & $7006 \ldots \ldots$ & -1.8 & 2,3 \\
$6171 \ldots \ldots$ & -0.9 & 1,5 & $7078 \ldots \ldots$ & -2.0 & 1,3 \\
$6205 \ldots \ldots$ & -1.4 & $1,3,4$ & $7089 \ldots \ldots$ & -1.4 & 1,3 \\
$6254 \ldots \ldots$ & -1.7 & 2,3 & $7099 \ldots \ldots$ & -2.0 & 1 \\
\hline
\end{tabular}

* Sources: 1. Butler 1975; 2. Canterna 1975; 3. Hesser, Hartwick, and McClure 1977; 4. Cohen 1978b; 5. Mould and McElroy 1978. 

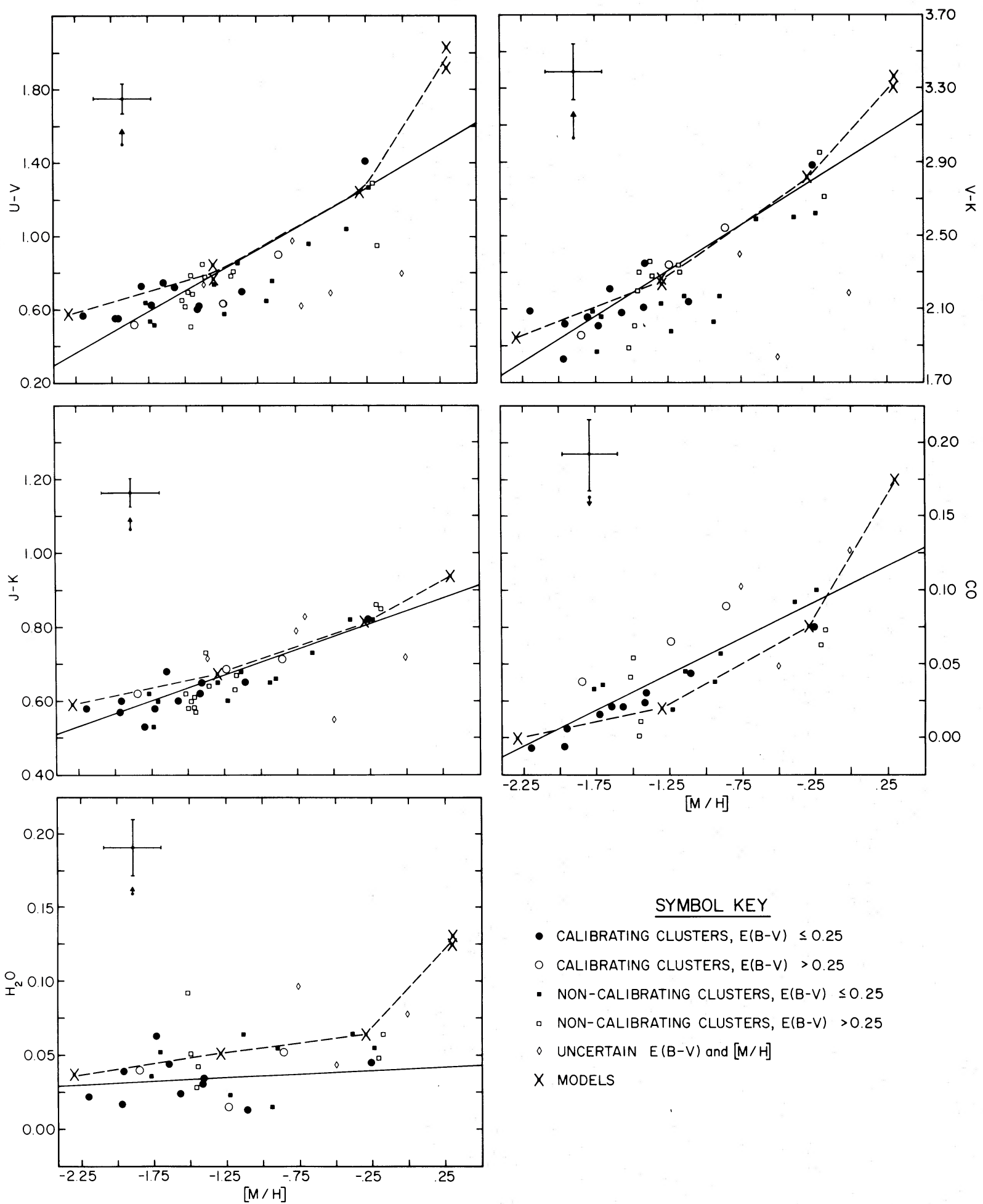

SYMBOL KEY

- calibrating clusters, e(B-V) $\leq 0.25$

- calibrating Clusters, E(B-V) $>0.25$

- NON-CALIBRATING CLUSTERs, E(B-V) $\leq 0.25$

- NON-CALIBRATING CLUSTERS, E(B-V) $>0.25$

- UNCERTAIN E(B-V) and [M/H]

$X$ MODELS

FIG. 1.-Various integrated globular cluster colors and indices are plotted against [M/H]. A least-squares line has been fitted through the calibrating clusters only, weighting the colors of the low-reddened clusters twice those of the high-reddened ones. The dashed line connects model results with $s=2.35$ from Table 3. The arrows indicate color change for $E(B-V)=0.05$. See text for further details. 
TABLE 2

Solutions to $Y=A+B * X$

\begin{tabular}{|c|c|c|c|c|c|c|c|c|}
\hline $\mathrm{N}$ & $x$ & $\mathrm{Y}$ & & A & & B & $r$ & $\mathrm{~s}_{\mathrm{y} / \mathrm{x}}$ \\
\hline $\begin{array}{l}14 \\
14 \\
14 \\
13 \\
13\end{array}$ & $\begin{array}{l}{[\mathrm{M} / \mathrm{H}]} \\
{[\mathrm{M} / \mathrm{H}]} \\
{[\mathrm{M} / \mathrm{H}]} \\
{[\mathrm{M} / \mathrm{H}]} \\
{[\mathrm{M} / \mathrm{H}]}\end{array}$ & $\begin{array}{c}U-V \\
V-K \\
J-K \\
\mathrm{CO} \\
\mathrm{H}_{3} \mathrm{O}\end{array}$ & $\begin{array}{l}1.39 \\
2.93 \\
0.85 \\
0.10 \\
0.04\end{array}$ & $\begin{array}{l}( \pm 0.11) \\
( \pm 0.11) \\
( \pm 0.03) \\
( \pm 0.01) \\
( \pm 0.01)\end{array}$ & $\begin{array}{l}0.45 \\
0.50 \\
0.14 \\
0.05 \\
0.01\end{array}$ & $\begin{array}{l}( \pm 0.07) \\
( \pm 0.07) \\
( \pm 0.02) \\
( \pm 0.01) \\
( \pm 0.01)\end{array}$ & $\begin{array}{l}0.84 \\
0.88 \\
0.88 \\
0.87 \\
0.17\end{array}$ & $\begin{array}{l}0.13 \\
0.13 \\
0.04 \\
0.01 \\
0.02\end{array}$ \\
\hline $\begin{array}{l}- \\
35 \\
35 \\
26 \\
26\end{array}$ & $\begin{array}{l}V-K \\
V-K \\
V=K \\
V=K\end{array}$ & $\begin{array}{c}U-V \\
J-K \\
\mathrm{CO} \\
\mathrm{H}_{2} \mathrm{O}\end{array}$ & $\begin{array}{r}-1.02 \\
-0.06 \\
-0.14 \\
0.01\end{array}$ & $\begin{array}{l}( \pm 0.14) \\
( \pm 0.06) \\
( \pm 0.03) \\
( \pm 0.03)\end{array}$ & $\begin{array}{l}0.80 \\
0.32 \\
0.08 \\
0.02\end{array}$ & $\begin{array}{l}( \pm 0.06) \\
( \pm 0.02) \\
( \pm 0.01) \\
( \pm 0.01)\end{array}$ & $\begin{array}{l}-- \\
0.89 \\
0.89 \\
0.72 \\
0.21\end{array}$ & $\begin{array}{l}0.10 \\
0.04 \\
0.02 \\
0.02\end{array}$ \\
\hline
\end{tabular}

allow for both uncertainties in $E(B-V)$ and possible variations in the reddening law itself (e.g., Racine 1973), the colors of clusters with $E(B-V)>0.25$ mag were given only half the weight of clusters with lower reddening values. The least-squares solutions from Figure 1 are summarized in the first part of Table 2 . These are essentially the same relations reported by Malkan, Aaronson, and Kleinmann (1977) for a smaller sample of clusters. We stress that because the solutions are heavily weighted by low-reddened clusters, any allowable variation in $E(B-V)$ or change in the reddening curve (Racine 1973) will not significantly affect the results.

Figure 1 and Table 2 show the well-known correlation of $U-V$ color with $[\mathrm{M} / \mathrm{H}]$. However, it is apparent that the $V-K$ and $J-K$ colors and the $\mathrm{CO}$ index also strongly correlate with metallicity.

The only other published survey of globular cluster infrared colors is that of Grasdalen (1974). He failed to detect a significant correlation between the $V-K$ color and metallicity, as measured by cluster spectral type, in his study of 14 compact, highly reddened clusters. In addition to the reddening uncertainties, Grasdalen's data suffered from both large corrections for reference beam flux and apparent instrumentation problems (see Frogel et al. 1975). In particular, the observed magnitudes at similar aperture sizes for clusters measured both by Grasdalen (1974) and Aaronson, Malkan, and Kleinmann (1978) are not in good agreement. Pritchet (1977) attempted to correct Grasdalen's data by adopting different reddening values. He found a resulting correlation between $V-K$ and metallicity which is consistent with the calibration adopted by Strom et al. (1976). As discussed below, the latter calibration does not adequately fit the present data.

The ratio $E(V-K) / E(B-V)=2.8$ given by the Whitford curve indicates the extreme sensitivity of the $V-K$ color to reddening. The correlation of $V-K$ with $[\mathrm{M} / \mathrm{H}]$ was also examined using the reddening values from Harris (1976) and from Burstein and McDonald (1975). While these correlations were essentially the same as in Table 2 , the points exhibited somewhat more scatter. Because the color excess values of Kron and Guetter (1976) were derived by forcing optical colors at a given spectral type to match, use of their reddening values will implicitly reduce the scatter in plots of optical data against metallicity. However, the scatter in plots of infrared data against $[\mathrm{M} / \mathrm{H}]$ will be reduced only if the infrared colors are themselves strongly correlated with $[\mathrm{M} / \mathrm{H}]$. In any event the ad hoc selection of reddening values for determination of $V-K$ color relations can be very misleading. We have adopted the uniformly determined reddening values of Kron and Guetter (1976) in order to avoid such bias.

Pritchet (1977) further argues that four clusters measured by Grasdalen (1974) are anomalous in the sense that their spectral types are too early for their $V-K$ colors, and he suggests that these clusters might have more blue horizontal-branch stars than normal. All four of these clusters were measured in the present work. The data for these objects fall close to the regression lines in Figure 1, and there does not appear to be evidence for such an anomalous effect.

In Figure 2 various colors have been plotted against $V-K$. The regression lines shown in Figure 2 and summarized in the bottom half of Table 2 were found by using all the clusters which have reddening values from Kron and Guetter (1976), but again the clusters with $E(B-V)>0.25$ were given half the weight of those with smaller color excesses. While all the scatter in $V-K$ in our figures might be accounted for by errors in $E(B-V)$, it is certainly no larger than the estimated error. A similar comment applies to the other colors and indices, as indicated by the variances listed in Table 2.

Also shown in Figure 2 are central region measurements of all early-type galaxies from Paper I, Paper II, and Aaronson (1978). In addition, measurements of three dwarf ellipticals have been taken from Persson, Frogel, and Aaronson (1978). The data are referred to a value of $\log A / D_{0}=-0.6$, where $A / D_{0}$ is the ratio of aperture size to corrected face-on diameter, the latter being adopted from de Vaucouleurs, de Vaucouleurs, and Corwin (1976). The well-defined sequence of galaxies with $V-K$ colors between 3.0 and 3.5 corresponds to an ordering of absolute magnitude $M_{V}$ from -19 to -23 (Paper I). The five galaxies with $V-K$ colors less than 3.0 are all dwarf 


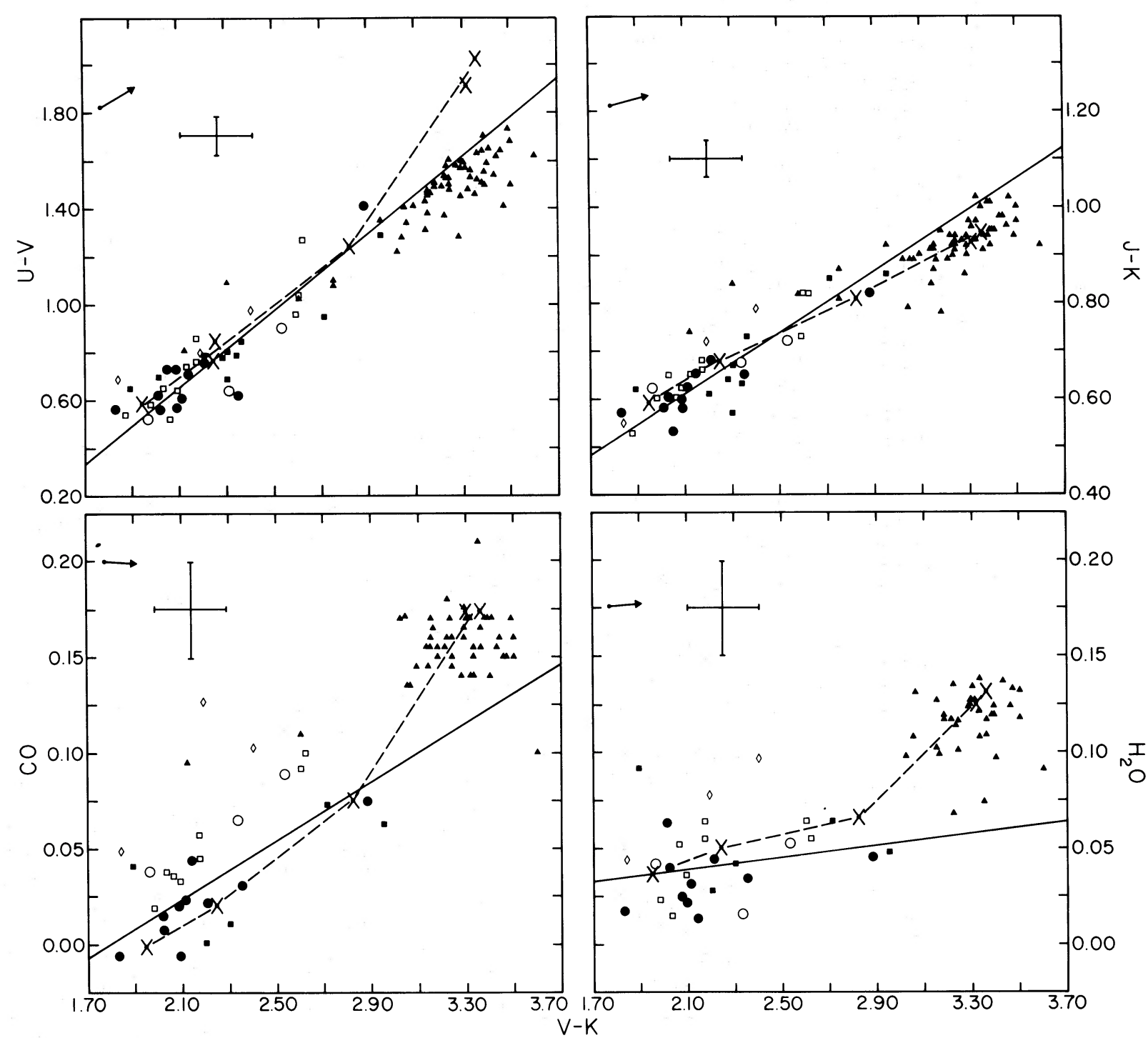

Fig. 2.-Globular cluster $U-V$ and $J-K$ colors, and $\mathrm{CO}$ and $\mathrm{H}_{2} \mathrm{O}$ indices, are plotted against $V-K$. Triangles, measurements of galaxies referred to an aperture size of $\log A / D_{0}=-0.6$. The other symbols have the same meaning as in Fig. 1. The leastsquares line has been fitted through all but those clusters with uncertain reddening and $[\mathrm{M} / \mathrm{H}]$, weighting the low-reddened points twice those with high reddening. The dashed line connects the model results with $s=2.35$ from Table 3 . The arrows indicate color change for $E(B-V)=0.05$. See text for further details.

ellipticals, which are again ordered by $M_{V}$ from -13 to -18 (Paper I; Persson, Frogel, and Aaronson 1978).

It is apparent from Figure 2 that in general the sequence defined by the galaxy points overlaps and extends the sequence of globular cluster points. Figure 2 suggests, from a purely empirical standpoint, that (1) the color-luminosity effect in galaxies is related to metallicity, and (2) the centers of bright ellipticals are metal-rich. McClure and van den Bergh (1968) and Faber $(1973,1977)$ have also discussed the empirical relation between globular clusters and galaxies and have reached similar conclusions.

\section{MODELS}

In order to study the extension of the globular cluster sequence in Figure 1 to elliptical galaxies, we need a physical model for integrated colors and indices in addition to a statistical model such as a least-squares extrapolation. To this end, we have followed the methods of Mould (1978) for construction of single-burst population models from the luminosity functions of $C D$. In these models the following additions and modifications were made to the CD data:

1. Inclusion of a core helium-burning stage. The strength of the horizontal branch (HB) is para- 
metrized by the ratio $n_{\mathrm{HB}} / n_{\mathrm{RG}}$ (the ratio of $\mathrm{HB}$ stars to red giants). The observed maximum of this ratio (which is theoretically controlled by the helium abundance) is 1.75 in 47 Tuc (Lee 1977), while a ratio of 0.8-0.9 typifies the metal-poor clusters (Iben et al. 1969). The need to include supra-HB ("UV-bright") stars suggested that this ratio be rounded to 1.0. To study the effects of different HB morphologies, we have considered three cases: (1) a blue HB covering the interval $(3.9,4.1)$ in $\log T_{e}$ (BHB), (2) a red $\mathrm{HB}$ extending 0.05 in $\log T_{e}$ blueward from the giant branch and offset from it by 0.02 (RHB), and (3) a clump on the giant branch with a width in $\log T_{e}$ of 0.02 .

2. An asymptotic giant branch. Above the HB, the first giant-branch luminosity function was multiplied by 1.22 (see Lee 1977). Unlike Tinsley and Gunn (1976b) and Tinsley (1977), we have not extended the giant branch above the red-giant tip. Observations of the clusters M3, M13, and M92 (Cohen, Frogel, and Persson 1978, hereafter CFP); M71 (Frogel, Persson, and Cohen 1978); and 47 Tuc (see Lloyd Evans and Menzies 1977) indicate that there are no stars more luminous than the theoretical first giant tip. This is probably a result of mass loss. This sample of globularcluster giants is larger and better defined than the old disk giants of Tinsley and Gunn (1976b), where the most luminous stars are all field Miras whose evolutionary status is less certain.

3. A lower main sequence. The $\mathrm{CD}$ isochrones were extended to a lower mass cutoff of $0.1 M_{\odot}$ following Mould (1978).

4. Modification of effective temperatures (cf. Demarque and McClure 1977). The effective temperature shift adopted by Mould (1978) was increased slightly to map the 13 billion-year isochrone $(Y=0.3$ and $Z=0.01$ ) onto a mean giant branch for 47 Tuc, weighted appropriately toward the asymptotic giants. This fit to a metal-rich cluster has the virtue of removing all but the differential errors in the temperature scale for red giants at the expense of introducing an uncertainty in the abundance calibration $( \pm 0.1 \mathrm{dex}$ for 47 Tuc; see McClure and Osborn 1974).

Information on colors and line indices was introduced into the population models from the following sources:

$V-K$. The model atmosphere calculations of CFP were adopted on the giant branch; elsewhere the calibration of Johnson (1966) was used without any metallicity dependence. Comparison of the shifted CD giant branch for $Z=0.0001$ with observations of M92 by CFP indicates that the former is systematically too red by $0.12 \mathrm{mag}$ in $V-K$. This error is propagated (to a reduced extent) into the integrated colors. By combining this with an uncertainty of 0.05 found by replacing the CD luminosity function with one observed for 47 Tuc (Da Costa 1977a), we estimate overall uncertainties of 0.15 in the model colors.

$U-V$. On the giant branch, an empirical relation between $U-V$ and $V-K$ as a function of temperature and metallicity was derived from the cluster observations of CFP. In the vicinity of the turnoff and main sequence, the ultraviolet excess calibration of Wallerstein (1962) was adopted and converted to $\Delta(U-V)$ by using the tabulation of Wildey $e t$ al. (1962). For the sake of comparison, further estimates of $\Delta(U-V)$ were made from $(U-V, R-I)$ relations for Eggen's (1977) moving groups. This yielded a slightly shallower relation between $U-V$ and metallicity, and gave integrated colors that were 0.07 redder at the metal-poor end and 0.03 bluer for $Z=0.04$. If the blanketing computations of Strom, Strom, and Carbon (1971) are used instead, a steeper relation results. We have adopted the original calibration of $U-V$, as the fit to the globular clusters is better. An uncertainty of $0.1 \mathrm{mag}$ in the model $U-V$ colors seems realistic.

$J-K . J-K$ was taken as a function of $V-K$ for giants and dwarfs from Paper I (corrected to the Johnson system) and from Johnson (1966) for the hotter stars. $J-K$ was assumed to be independent of $Z$ based on the results of CFP. The dominant uncertainty in the calculated integrated $J-K$ colors arises from relative errors in the temperature shift of the $C D$ giant branch as a function of $Z$ and is onethird of the uncertainty in $V-K$, or $0.05 \mathrm{mag}$.

CO. Carbon monoxide as a function of $V-K$ for solar-metallicity dwarfs and giants was taken from Paper I. The change in the photometric CO index with metallicity, due to column density and saturation effects, was expressed (as in Cohen 1978a) by $A(\mathrm{CO}) \propto$ $Z^{\mathrm{MMF}}$, where $A(\mathrm{CO})$ is the absorption percentage represented by the $\mathrm{CO}$ index. This function was derived by integrating the opacity distribution function (ODF) spectra $^{2}$ in the $2.4 \mu \mathrm{m}$ region from the Bell et al. (1976) and Gustafsson et al. (1975) grids of models for $\mathrm{K}$ giants, and it satisfactorily reproduces the cluster observations of CFP. The same curve was assumed to hold for $M$ giants, with a minimum value for $\mathrm{MMF}(\mathrm{CO})$ of 0.1 . Carbon monoxide indices for dwarfs were not corrected for metallicity effects unless they were larger than $0.03 \mathrm{mag}$. Throughout these calculations, the appropriate corrections were made to account for the single-sideband nature of the observed CO index. At constant $T_{\text {eff }}$, the ODF spectra did not predict any variation of the $\mathrm{CO}$ absorption with surface gravity within the range expected for giants. The predicted $\mathrm{CO}$ indices are expected to be accurate to $\pm 0.02 \mathrm{mag}$, based on calculations with $\mathrm{MMF}(\mathrm{CO})$ curves that vary from those predicted by the ODF spectra.

$\mathrm{H}_{2} \mathrm{O}$. The $\mathrm{H}_{2} \mathrm{O}$ index as a function of $V-K$ for solar metallicity giants and dwarfs was taken from Paper II. FTS spectra of bright $\mathrm{K}$ and $\mathbf{M}$ giants provided by Ridgway (1977) clearly demonstrate that the $\mathrm{H}_{2} \mathrm{O}$ band consists of many weak lines, which first become visible between spectral types M2 and M4. The calculations of Goon and Auman (1970) predict that the column density of $\mathrm{H}_{2} \mathrm{O}$ above $\tau_{2.7 \mu \mathrm{m}}=0.3$ is independent of metallicity, but depends strongly on surface gravity. The resulting impact on the $\mathrm{H}_{2} \mathrm{O}$

${ }^{2}$ The ODF fluxes for these models were kindly provided by Dr. Bengt Gustafsson. 
photometric index of variation of $\mathrm{H}_{2} \mathrm{O}$ column density will be large, as the $\mathrm{H}_{2} \mathrm{O}$ lines are mostly on the linear part of the curve of growth; this is also implied by the rapid increase in the photometric $\mathrm{H}_{2} \mathrm{O}$ index in stars cooler than M3. Assuming that many weak lines form the $\mathrm{H}_{2} \mathrm{O}$ band, we have used the column density calculations to derive values of $\mathrm{MMF}\left(\mathrm{H}_{2} \mathrm{O}\right)$ from the predicted change of gravity with $Z$ for giants at fixed $T_{\text {eff }}$. (The observations of globular-cluster red giants in CFP do not reach stars cool enough to determine empirically the correct $\mathrm{MMF}$ for $\mathrm{H}_{2} \mathrm{O}$.) Appropriate corrections for the single-sideband nature of the $\mathrm{H}_{2} \mathrm{O}$ index have also been made. These calculations are most uncertain $( \pm 0.04 \mathrm{mag})$ for the metal-rich case, although less uncertain $( \pm 0.02 \mathrm{mag})$ for the metalpoor cases (where the predicted $\mathrm{H}_{2} \mathrm{O}$ index is due almost entirely to the continuum slope rather than the actual presence of $\mathrm{H}_{2} \mathrm{O}$ spectral features), and are meant as an illustrative guide to the expected behavior. Both the $\mathrm{H}_{2} \mathrm{O}$ and $\mathrm{CO}$ calculations assume uniform metallicity variations, i.e., a constant $\mathrm{C} / \mathrm{O}$ ratio.

Colors for populations of age $13 \times 10^{9}$ years with $Y=0.3$ are presented in Table 3. Models with $s=$ 2.35 are plotted in Figures 1 and 2 .

\section{DISCUSSION}

\section{a) Globular Clusters}

As discussed in $\S$ II, Figures 1 and 2 clearly reveal that globular clusters form well-defined metallicity sequences in $V-K, U-V, J-K$, and $\mathrm{CO}$, and that in two-color diagrams the central regions of elliptical galaxies lie along and extend the sequences defined by the globular clusters. The agreement between the globular cluster observations and the simple models of single-burst star formation $13 \times 10^{9}$

TABLE 3

Colors and Indices of the Population Models*

\begin{tabular}{|c|c|c|c|c|c|c|}
\hline $\begin{array}{c}\mathrm{M} / \mathrm{H}] \\
\mathrm{HB} \\
n_{\mathrm{HB}} / n_{\mathrm{RG}}\end{array}$ & $s$ & $U-V$ & $V-K$ & $J-K$ & $\mathrm{CO}$ & $\mathrm{H}_{2} \mathrm{O}$ \\
\hline $\begin{array}{r}-2.3 \\
\text { BHB } \\
1.0\end{array}$ & $\begin{array}{c}0 \\
2.35 \\
4\end{array}$ & $\begin{array}{l}0.57 \\
0.58 \\
0.69\end{array}$ & $\begin{array}{l}1.82 \\
1.94 \\
2.69\end{array}$ & $\begin{array}{l}0.53 \\
0.59 \\
0.77\end{array}$ & $\begin{array}{r}0.002 \\
-0.001 \\
-0.019\end{array}$ & $\begin{array}{l}0.030 \\
0.036 \\
0.060\end{array}$ \\
\hline $\begin{array}{r}-1.3 \\
\text { BHB } \\
1.0\end{array}$ & $\begin{array}{c}0 \\
2.35 \\
4\end{array}$ & $\begin{array}{l}0.77 \\
0.77 \\
0.87\end{array}$ & $\begin{array}{l}2.16 \\
2.24 \\
2.93\end{array}$ & $\begin{array}{l}0.64 \\
0.68 \\
0.81\end{array}$ & $\begin{array}{r}0.030 \\
0.020 \\
-0.010\end{array}$ & $\begin{array}{l}0.043 \\
0.050 \\
0.079\end{array}$ \\
\hline $\begin{array}{r}-1.3 \\
\text { RHB } \\
1.0\end{array}$ & $\begin{array}{c}0 \\
2.35 \\
4\end{array}$ & $\begin{array}{l}0.84 \\
0.84 \\
0.92\end{array}$ & $\begin{array}{l}2.19 \\
2.26 \\
2.94\end{array}$ & $\begin{array}{l}0.64 \\
0.67 \\
0.80\end{array}$ & $\begin{array}{r}0.029 \\
0.019 \\
-0.010\end{array}$ & $\begin{array}{l}0.042 \\
0.049 \\
0.079\end{array}$ \\
\hline $\begin{array}{c}-0.3 \\
\text { RHB } \\
1.75\end{array}$ & $\begin{array}{c}0 \\
2.35 \\
4\end{array}$ & $\begin{array}{l}1.24 \\
1.24 \\
1.37\end{array}$ & $\begin{array}{l}2.80 \\
2.82 \\
3.42\end{array}$ & $\begin{array}{l}0.82 \\
0.81 \\
0.86\end{array}$ & $\begin{array}{l}0.100 \\
0.075 \\
0.008\end{array}$ & $\begin{array}{l}0.053 \\
0.064 \\
0.126\end{array}$ \\
\hline $\begin{array}{l}0.3 \\
\text { RHB } \\
1.75\end{array}$ & $\begin{array}{c}0 \\
2.35 \\
4\end{array}$ & $\begin{array}{l}1.97 \\
1.92 \\
1.95\end{array}$ & $\begin{array}{l}3.34 \\
3.31 \\
3.80\end{array}$ & $\begin{array}{l}0.95 \\
0.93 \\
0.95\end{array}$ & $\begin{array}{l}0.200 \\
0.173 \\
0.070\end{array}$ & $\begin{array}{l}0.087 \\
0.100 \\
0.211\end{array}$ \\
\hline $\begin{array}{c}0.3 \\
\text { clump } \\
1.75\end{array}$ & $\begin{array}{c}0 \\
2.35 \\
4\end{array}$ & $\begin{array}{l}2.11 \\
2.03 \\
2.05\end{array}$ & $\begin{array}{l}3.40 \\
3.36 \\
3.84\end{array}$ & $\begin{array}{l}0.96 \\
0.94 \\
0.96\end{array}$ & $\begin{array}{l}0.202 \\
0.175 \\
0.072\end{array}$ & $\begin{array}{l}0.093 \\
0.104 \\
0.212\end{array}$ \\
\hline
\end{tabular}

years ago, with $s=2.35$ (the Salpeter IMF) and varying $Z$, is remarkable. The $\mathrm{CO}-[\mathrm{M} / \mathrm{H}]$ diagram for globulars demonstrates a strong correlation, particularly for the low-reddened calibrating clusters.

For globular clusters, models with substantial contributions to the integrated light by $\mathbf{M}$ dwarfs (i.e., those with $s \gtrsim 4$ ) can be eliminated, as they produce colors which are too $\mathrm{red}^{3}$ for a given $[\mathrm{M} / \mathrm{H}]$, $\mathrm{H}_{2} \mathrm{O}$ indices which are too large, and $\mathrm{CO}$ indices which are too small. This claim can be made only for the present-day mass function of the inner regions of the clusters observed. In 47 Tuc, Da Costa (1977b) has found that $3.4<s<4.4$ for the cluster as a whole from star counts and a dynamical model. However, the mass segregation he observes in 47 Tuc is predicted to restrict $s$ below 2 in the inner $2^{\prime}$. Unfortunately, with the present observational data, the colors (Table 3) are insufficiently sensitive to choose between IMFs with $s \leq 2.35$.

Although observational uncertainties seem capable of explaining most of the scatter in Figures 1 and 2, two effects which will intrinsically spread the integrated colors deserve comment. First, we have investigated the stochastic effect on $U-V$ and $V-K$ of the finite population of stars in a globular cluster. A Monte Carlo simulation of the brightest 60,000 stars in a cluster of $M_{V}=-7.5$ gave colors differing from the appropriate continuous model $([\mathrm{M} / \mathrm{H}]=$ $-0.3, s=2.35$ ) by $\sigma=0.10$ in $V-K$ and 0.015 in $U-V$. Such a model is close to the mean luminosity of globulars, which have a dispersion in absolute magnitude of approximately $1 \mathrm{mag}$ (see Hanes 1977). A cluster 4 times less luminous yielded a color dispersion twice as large. It is clear that the considerably greater predicted dispersion in $V-K$ stems from the much smaller sample of stars contributing to the $2 \mu \mathrm{m}$ integrated light. The stochastic effects on the $\mathrm{H}_{2} \mathrm{O}$ index will also be large. As the clusters of the present sample tend to be brighter than the mean, stochastic scatter in $V-K$ is masked by observational scatter; however, the size of the predicted effect should be kept in mind for the interpretation of sparse clusters.

A second source of intrinsic scatter is the unsystematic behavior of the HB. Although the general trend of a reddening $\mathrm{HB}$ with rising metallicity and increasing $n_{\mathrm{HB}} / n_{\mathrm{RG}}$ is well known, red and blue HBs can be found at similar $[\mathrm{M} / \mathrm{H}]$, especially in clusters of intermediate metallicity. The effect of a change from blue to red $\mathrm{HB}$ can be seen in the $(U-V, V-K)$ diagram of Figure 2 . As $U-V$ is much more sensitive to $\mathrm{HB}$ light than $V-K$, the displacement is well away from the metallicity and reddening lines. For the lowreddened objects of intermediate metallicity in this diagram, there is a fairly clear tendency for clusters with HB morphology greater than Dickens (1972) class 3 to lie above the mean line and Dickens class

\footnotetext{
${ }^{3}$ If $s=4$, the $V-K$ color is sensitive to the lower mainsequence termination, here assumed to be $0.1 M_{\odot}$ (Graboske and Grossman 1971). If this value is changed to $0.05 M_{\odot}$, $V-K$ increases by 0.5 mag. Similar sensitivity is expected in the $\mathrm{H}_{2} \mathrm{O}$ and $\mathrm{CO}$ indices. For $s \leq 2.35$, no effect is found, as the $2 \mu \mathrm{m}$ light is then giant dominated.
} 
2 or less to lie below the mean line. The mean amplitude of the separation is close to that of the models; and to correct the impression given by Pritchet (1977), we stress its moderate size.

\section{b) Elliptical Galaxies}

Since the globular cluster observations are consistent with our model using the Salpeter IMF, we now use the models to consider what observations of the same photometric quantities imply regarding the centers of elliptical galaxies. Figure 2 and Table 3 demonstrate that, with the exception of $U-V$ (see below), observations of early-type galaxies with $-19 \geq M_{V} \geq-23$ can be fitted by models with $s=$ 2.35 and metallicity index ${ }^{4}[\mathrm{M} / \mathrm{H}]$ ranging from 0.0 to +0.3 . In particular, giant elliptical galaxies appear metal-rich with $[\mathbf{M} / \mathbf{H}] \approx 0.3$; this result agrees with estimates by Faber (1973) and Cohen (1978a). The dwarf ellipticals with $M_{V}>-18$ would appear to have $[\mathrm{M} / \mathrm{H}]<0$. Note that while models with $s<2.35$ are equally acceptable, models with $s=4$ can be ruled out.

A proper consideration of the change in the photometric CO index with metallicity ( $\$$ III) shows that $s=2.35$ can easily explain the observed mean $\mathrm{CO}$ index for giant elliptical galaxies of approximately $0.16 \mathrm{mag}$, as opposed to the results (see below) of Tinsley and Gunn (1976) and Tinsley (1977). We cannot, of course, use the $\mathrm{CO}$ index to derive values of $s$ for individual galaxies because of both the weak sensitivity of the feature for low values of $s$ and the accumulated uncertainties. Nevertheless, we can use the location of galaxies in the $(\mathrm{CO}, V-K)$-diagram to place an upper limit on $s$ : in this diagram the loci of increasing $s$ and of increasing $[\mathrm{M} / \mathrm{H}]$ are orthogonal. A crude interpolation between our three available IMFs leads to an upper limit on $s$ of 3.2.

The calculated model $\mathrm{H}_{2} \mathrm{O}$ index is also consistent with the mean for giant ellipticals without departure from the Salpeter IMF. The strong increase in the $\mathrm{H}_{2} \mathrm{O}$ index between $[\mathrm{M} / \mathrm{H}]=-0.3$ and +0.3 is due to the absence of $\mathrm{M}$ giants later than M5 from the CD tracks for $[\mathrm{M} / \mathrm{H}] \leq-0.3$. The presence of late $\mathrm{M}$ giants (contributing approximately $27 \%$ of the light at $K)$, in the case where $[\mathrm{M} / \mathrm{H}]=+0.3$, supports the conclusions of Paper II.

Models with $[\mathrm{M} / \mathrm{H}]=0.3$ do not fit the observed galaxy colors in $U-V$. The discrepancy is considerably larger than the estimated uncertainty in the model colors of $0.1 \mathrm{mag}$. A similar problem was encountered by Tinsley (1977), who suggests the presence of blue stragglers, or a minority of young or metal-poor stars, as possible solutions. A strong

${ }^{4}$ The galaxy data have been corrected for reddening and redshift (Papers I and II). The reddening correction used is the absorption-free polar-cap model of Sandage (1973); the mean galactic latitude of the galaxy sample is $\left\langle b^{\mathrm{II}}\right\rangle \sim 55^{\circ}$. If nonzero reddening is present at the pole (Burstein and McDonald 1975; Kron and Guetter 1976), the abundance estimate in galaxies from their $V-K$ colors should be adjusted downward. For instance, a zero-point shift of 0.03 in $E(B-V)$ leads to a shift of $\Delta[\mathrm{M} / \mathrm{H}] \approx-0.1$, for $s=2.35$. blue horizontal branch would certainly be effective in this regard. Indeed, a prototype for such a system, with a spread in metallicity but a single age, may already exist in $\omega$ Cen (Freeman and Rodgers 1975). Because the light from metal-poor (blue) stars dominates $U-V$, but that from metal-rich (red) stars dominates $V-K$, two-component models constructed from Table 3 depart from the locus of homogeneous models in Figure 2 and can match the colors of bright ellipticals. For example, the colors of a model with $20 \%$ of the visual light from a population with $[\mathrm{M} / \mathrm{H}]=-2.3$ and $80 \%$ from a population with $[\mathrm{M} / \mathrm{H}]=0.3$ are $U-V=1.49$ and $V-K=3.15$.

Another plausible possibility is that the abundance of iron-peak elements, which drive the ultraviolet deficiency, is not enhanced to the extent of the light elements, which control the photospheric opacity. Light elements such as $\mathrm{Na}, \mathrm{Mg}$, and $\mathrm{Si}$ provide most of the electrons to make up the $\mathrm{H}^{-}$opacity, and others such as $\mathrm{C}, \mathrm{N}$, and $\mathrm{O}$ provide the molecular contribution. The photospheric opacity, in turn, controls the position of the giant branch (Demarque and McClure 1977). Evidence from the $\lambda 5270$ blend that $\mathrm{Fe}$ does not vary between giant and dwarf ellipticals in step with $\mathrm{Na}, \mathrm{Mg}$, and $\mathrm{CN}$ has been presented by Faber (1977; see also remarks by Mould 1978, and Peterson 1976). A quantitative test of this proposal was performed on a model with $[\mathrm{M} / \mathrm{H}]=$ +0.3 and a red horizontal branch by holding $\Delta(U-V)$ at the value for solar composition. The resultant integrated color was $U-V=1.77$ with no change in $V-K$. This value approaches that of giant ellipticals within the range of uncertainty of the calculated colors.

To test the sensitivity of the model colors to age effects, we have calculated a younger model with age 6 billion years and $Z=0.01$. Its colors were bluer by 0.22 in $U-V$ and 0.28 in $V-K$ as a result of the hotter turnoff and hotter giant branch. Since age and composition differences are inseparable in the broadband colors, for the present purposes we assume that the age of elliptical galaxies is the same as that of globular clusters. We note that a difference of 3 billion years would affect our composition estimates by $\sim 0.1$ in $[\mathrm{M} / \mathrm{H}]$

The mass-to-light ratios for the luminous component of the models are only weakly composition dependent and are approximately $0.7,4$, and 50 for $s=0,2.35$, and 4 , respectively. For the flat mass function $(s=0)$, remnant corrections are large (and poorly defined); for $s>2$, corrections for fragments (black dwarfs with masses lower than the mainsequence termination) $m a y^{5}$ be considerable. For

\footnotetext{
${ }^{5}$ The turnover in Luyten's (1968) luminosity function apparently before the main-sequence termination point $m_{\mathrm{H}}$ suggests that the minimum mass for fragments $m_{\mathrm{L}}$ may not be very different in the solar neighborhood from $m_{\mathrm{H}}$. However, the existence of the turnover remains open to question (see Weistrop 1978), and theoretical values of $m_{\mathrm{L}}$ as low as $0.007 M_{\odot}$ have been discussed (Low and Lynden-Bell 1976). Note that in the solar neighborhood, at least, the combined remnant and fragment corrections cannot exceed a factor of 3 (see Wielen 1974).
} 
these reasons, mass-to-light ratios cannot be used to decisively constrain the models.

With the assumption of Salpeter mass functions, color-magnitude relations, such as those in Paper I and in Visvanathan and Sandage (1977), can be calibrated in terms of metallicity by using Table 3 . For example, the $V-K$ observations of Paper I yield $\Delta(V-K) / \Delta M_{V} \approx-0.07$, and therefore $\Delta \log Z \mid$ $\Delta M_{V} \approx-0.08$. Because of the discrepancy discussed above, caution should be used in applying the model results for $U-V$.

It is often argued (e.g., Strom et al. 1976, 1978) that color changes within early-type galaxies have the same cause as color changes among such galaxies. However, the data that exist in this regard are ambiguous (Paper I; Persson, Frogel, and Aaronson 1978), and the possibility, for instance, of selective elemental enhancement within galaxies (Peterson 1976; Faber 1977) cannot be ruled out. Nevertheless, as a first approximation, the results in Table 3 should also be useful for calibrating color-aperture effects with $[\mathrm{M} / \mathrm{H}]$.

\section{c) Comparison with Other Models}

We now compare our conclusions on $[\mathrm{M} / \mathrm{H}]$ and $s$ in bright ellipticals with those of previous investigations. First, we note that the $(V-K, \log Z)$ calibration given by Strom et al. (1976) essentially agrees with our models both in the zero point $(V-K$ at solar abundance ${ }^{6}$ ) and slope for $[\mathrm{M} / \mathrm{H}] \geq-0.3$. Their slope, however, cannot be extrapolated to more metal-poor systems, as the mean fit to the globulars (Table 2) and the metal-poor models (Table 3 ) indicate that a much smaller slope is appropriate. We also note that this agreement of the zero point is fortuitous, as use of the $Z=0.01$ track of Rood (1972) by Strom et al. (1976) for solar abundance $(Z=0.02)$ happens to provide the appropriate temperature modification to the Rood giant branch (see $\S$ III, point 4).

Tinsley and Gunn (1976a) obtain $s<2.0$ from observations of the $\mathrm{CO}$ index and from the $\mathrm{FeH}$ observations of Whitford (1977), which, however, have been disputed by Cohen (1978). Their reliance on the nondetection of $\mathrm{FeH}$ in galaxies led them to conclude that $s$ must be significantly less than the Salpeter value. Tinsley (1977) attempts to extend the Tinsley-Gunn models to include metallicity variations. She constructs models at $Z=0.01$ and $Z=0.04$ by using the $C D$ tracks for the main sequence and subgiant branch, but she does not do so for the giant branch. Instead, she introduces ad hoc extra $M$ giants in order to produce the mean observed elliptical $V-K$ color and $\mathrm{H}_{2} \mathrm{O}$ index. In particular, with the low metallicity $(Z=0.01)$ adopted in Tinsley's (1977) standard composition model, the predicted value of the $\mathrm{CO}$ index can only be made large enough to reach that of the giant ellipticals by making $s$ very small $(s<2.0$ or $x<1.0)$. However, the colors and indices of Tinsley's (1977) $Z=0.01$ model are in

${ }^{6}$ Lambert's (1976) revised solar abundances yield $Z_{\odot}=0.02$. direct disagreement with the empirical results in Figure 1 . For this metallicity, we expect a $V-K$ color $\approx 2.9$, a $\mathrm{CO}$ index $\approx 0.08$, and an $\mathrm{H}_{2} \mathrm{O}$ index $\approx 0.05$, whereas Tinsley (1977) finds values of $3.30,0.15$, and 0.13 , respectively, for these three quantities (her Table 1). For similar reasons, the $V-K$ color in her $Z=0.04$ model is also substantially redder than that in our model. It is interesting to note that the models in Tinsley and Gunn (1976a), which are, perhaps, more directly related to nearby, slightly metal-poor, old disk stars than those of Tinsley (1977), give results closer to the empirical values at $Z=0.01$. For example, their model A yields $2.97,0.14$, and 0.08 for $V-K, \mathrm{CO}$, and $\mathrm{H}_{2} \mathrm{O}$, respectively.

Tinsley (1977) further finds that $\Delta(V-K)$ $\Delta \log Z \approx 0.6$ (between $Z=0.01$ and 0.04 ) compared with our value of $0.8-0.9$. Thus, by using the results in Paper I, she derives $\Delta \log Z \mid \Delta M_{V} \approx-0.12$, which is $50 \%$ larger than our estimate in $\S \mathrm{IV} b$. In addition, her models predict a color ratio change $\Delta(V-K)$ / $\Delta(B-V) \approx 1.4$, and she argues that this value agrees with the ratio obtained from empirical $B-V$ and $V-K$ color-magnitude diagrams. However, Sandage (1972) has stressed the weakness of the color-magnitude relation in $B-V$. For the globular cluster sample of this paper, the correlation between $B-V$ and $[\mathrm{M} / \mathrm{H}]$ (for which $r=0.77$ ) is poorer than for either $U-B(r=0.87)$ or $U-V(r=0.84)$. It seems clear that $B-V$ is not the best measure of metallicity, although the relationship between $Z$ and galactic mass of Tinsley (1977) is not greatly changed by using $V-K$ instead.

Recognition that giant ellipticals have $[\mathrm{M} / \mathrm{H}] \approx$ +0.3 and correction of the computed colors for the enhanced metallicity removes the requirement of small $s$ values. We believe that our result $(s<3.2$ and enhanced metallicity) is more internally consistent and in better accord with known data on globular clusters and ellipticals than Tinsley's (1977) treatment of the giant branch, i.e., the ad hoc addition of $\mathbf{M}$ giants. We emphasize that models with $s=2.35$ and $[\mathrm{M} / \mathrm{H}]$ between 0.0 and +0.3 successfully reproduce all observed photometric indices (except $U-V$ ) for bright elliptical galaxies, and that models with the same $s$ but lower metallicities reproduce observed photometric indices for the globular clusters.

\section{CONCLUSIONS}

The conclusions of this work can be summarized as follows:

1. A strong correlation has been established between the metallicity of a large sample of globular clusters and their integrated infrared colors. This correlation is seen in both the $\mathrm{CO}$ index and broadband $V-K$ and $J-K$ colors.

2. The location of the centers of early-type galaxies in two-color diagrams forms an extension of the globular cluster sequence, and suggests that galaxies ordered by absolute magnitude are also ordered by metallicity, with the brightest ellipticals being metalrich. 
3. A calibration of integrated colors for composite stellar systems in terms of metallicity has been derived by using population models which incorporate a careful treatment of composition effects. These models should provide a better calibration of the colors than a simple extrapolation of the globular cluster relations, as the color-metallicity effects are predicted to be nonlinear. The good fit of the models to the globular clusters gives us confidence in the reliability of the calibration. In particular, the present models adequately reproduce empirical colors for $Z=0.01$, in marked contrast to the models of Tinsley (1977). We note, however, that early-type galaxies have an ultraviolet excess relative to models with a metallicity suggested by the infrared colors. A spread in composition may be of importance in explaining this discrepancy.

4. While in the present paper we have assumed that ellipticals and globular clusters have the same age, differences in age of order $3 \times 10^{9}$ years can mimic composition differences of $0.1 \mathrm{in}[\mathrm{M} / \mathrm{H}]$.

5. The colors at a given $[\mathrm{M} / \mathrm{H}]$ are fairly insensitive to the IMF for a power-law slope $s \leq 2.35$. However, proper accounting of metallicity effects on the $\mathrm{CO}$ and $\mathrm{H}_{2} \mathrm{O}$ indices permits a steeper IMF than implied by earlier work (cf. Tinsley and Gunn 1976; Tinsley 1977). Galaxies which have $-19 \geq M_{\mathrm{V}} \geq-23$ can be fitted by a range in metallicity of $0.0 \leq[\mathrm{M} / \mathrm{H}] \leq$ +0.3 with $s=2.35$. Values of $s<2$ cannot be ruled out; however, an upper limit of $s=3.2$ does seem necessary. Thus the evolutionary luminosity corrections may not be as large as suggested by the work of Tinsley $(1972,1977)$.

We thank John Huchra, Bruce Carney, and Phil Marcus for their assistance at the telescope. We also thank Doug Kleinmann, Jay Frogel, Eric Persson, and George Rieke for discussions and encouragement during the course of this work. M. A. and M. M. thank the Center for Astrophysics for generous financial support. This research was partially supported with funds from NSF grants AST 76-81874 and AST 7622991.

\section{REFERENCES}

Aaronson, M. 1978, in preparation.

Aaronson, M., Frogel, J. A., and Persson, S. E. 1978, Ap. J., 220, 442 (Paper II).

Aaronson, M., Malkan, M., and Kleinmann, D. 1978, in preparation.

Baldwin, J. R., Frogel, J. A., and Persson, S. E. 1973, Ap. J., $184,427$.

Bell, R. A., Eriksson, K., Gustafsson, B., and Nordlund, A. 1976, Astr. Ap., 23, 37.

Burstein, D., and McDonald, L. H. 1975, A.J., 80, 17.

Butler, D. 1975, Ap.J., 200, 68.

Canterna, R. 1975, Ap. J. (Letters), 200, L63.

Ciardullo, R. B., and Demarque, P. 1977, Trans. Astr. Obs., Yale University, Vol. 35 (CD).

Cohen, J. G. 1978a, Ap. J., 221, 788. $1978 b, A p . J$. in press.

Cohen, J. G., Frogel, J. A., and Persson, S. 1978, Ap. J., 222 , 165 (CFP).

Da Costa, G. S. 1977a, private communication.

. 1977b, Ph.D. thesis, Australian National University.

Demarque, P., and McClure, R. D. 1977, Ap. J., 213, 716.

de Vaucouleurs, G., de Vaucouleurs, A., and Corwin, H. G. 1976, Second Reference Catalogue of Bright Galaxies (Austin: University of Texas Press)

Dickens, R. J. 1972, M.N.R.A.S., 157, 281.

Eggen, O. J. 1977, Ap. J., 215, 812.

Faber, S. M. 1972, Astr. Ap., $20,361$. 1973, Ap. J., 179, 731.

. 1977, in The Evolution of Galaxies and Stellar Populations, ed. B. M.. Tinsley and R. B. Larson (New Haven: Yale University Observatory), p. 157.

Freeman, K. C., and Rodgers, A. W. 1975, Ap. J. (Letters), 201, 71 .

Frogel, J. A., Persson, S. E., Aaronson, M., Becklin, E. E., Matthews, K., and Neugebauer, G. 1975, Ap. J. (Letters), 200, L123.

Frogel, J. A., Persson, S. E., Aaronson, M., and Matthews, K. 1978, Ap. J., 220, 75 (Paper I)

Frogel, J. A., Persson, S. E., and Cohen, J. G. 1978, in preparation.

Goon, G., and Auman, J. R. 1970, Ap. J., 161, 533.

Graboske, H. C., and Grossman, A. 1971, Ap. J., 170, 363.

Grasdalen, G. L. 1974, A.J., 79, 1047.

Gustafsson, B., Bell, R. A., Eriksson, K., and Nordlund, A. 1975, Astr. Ap., 42, 407.

Hanes, D. A. 1977, M.N.R.A.S., 180, 309.

Harris, W. E. 1976, A.J., 81, 1095.
Harris, W. E., and van den Bergh, S. 1974, A.J., 79, 31.

Hesser, J. E., Hartwick, F. D. A., and McClure, R. D. 1977, Ap. J. Suppl., 33, 471.

Iben, I., Rood, R. T., Strom, K. M., and Strom, S. E. 1969, Nature, 224, 1006.

Johnson, H. L. 1966, Ann. Rev. Astr. Ap., 4, 192.

. 1968, in Stars and Stellar Systems, Vol. 7, ed. B. M. Middlehurst and L. H. Aller (Chicago: University of Chicago Press), p. 167

King, I. R. 1966, A.J., 71, 64

Kron, G. E., and Guetter, H. H. 1976, A.J., 81, 817.

Kron, G. E., and Mayall, N. 1960, A.J., 65, 581.

Kukarkin, B. 1975, Globular Star Clusters (Washington: NASA Technical Translation).

Lambert, D. L. 1976, Trans. IAU, 16B, 114

Lee, S.-W. 1977, Astr. Ap. Suppl., 27, 381.

Lloyd Evans, T., and Menzies, J. W. 1977, M.N.R.A.S., 178, 163.

Low, C., and Lynden-Bell, D. 1976, M.N.R.A.S., 176, 367.

Luyten, W. J. 1968, M.N.R.A.S., 139, 221.

Malkan, M., Aaronson, M., and Kleinmann, D. 1977, Bull. $A A S, 9,294$.

McClure, R. D., and Osborn, W. 1974, Ap. J., 189, 405.

McClure, R. D., and van den Bergh, S. 1968, A.J., 73, 313.

Mould, J. R. 1978, Ap. J., 220, 434.

Mould, J. R., and McElroy, D. B. 1978, Ap. J., 220, 935.

O'Connell, R. W. 1976, Ap. J., 106, 370.

Persson, S. E., Frogel, J. A., and Aaronson, M. 1978, in preparation.

Peterson, C., and King, I. 1975, A.J., 80, 427

Peterson, R. C. 1976, Ap. J. (Letters), 210, L123.

Pritchet, C. 1977, A.J., 82, 471.

Racine, R. 1973, A.J., 78, 180.

Ridgway, S. 1977, private communication.

Rood, R. T. 1972, Ap. J., 177, 681.

Sandage, A. 1972, Ap. J., 176, 21

-. 1973, Ap. J., 183, 711.

Sandage, A., and Widley, R. 1967, Ap. J., 150, 469.

Strom, K. M., Strom, S. E., Wells, D. C., and Romanishin, W. 1978, Ap. J., 220, 62.

Strom, S. E., Strom, K. M., and Carbon, D. F. 1971, Astr. Ap., $12,177$.

Strom, S. E., Strom, K. M., Goad, J. W., Vrba, F. J., and Rice, W. 1976, Ap. J., 204, 684.

Tinsley, B. M. 1972, Ap. J., 178, 319.

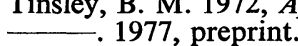


Tinsley, B. M., and Gunn, J. E. 1976a, Ap. J., 203, 52. $1976 b, A p . J ., 206,525$.

Visvanathan, N., and Sandage, A. R. 1977, Ap. J., 216, 214.

Wallerstein, G. 1962, Ap. J., Suppl., 6, 407.

Weistrop, D. 1978, in preparation.
Whitford, A. E. 1977, Ap. J., 211, 527.

Wielen, R. 1974, in Highlights of Astronomy, Vol. 3, ed. G. Contopoulos (Dordrecht: Reidel), p. 395.

Wildey, R. L., Burbidge, E. M., Sandage, A. R., and Burbidge, G. R. 1962, Ap. J., 135, 94.

MARC Aaronson: Steward Observatory, University of Arizona, Tucson, AZ 85721

Judith G. Cohen and Jeremy Mould: Kitt Peak National Observatory, P.O. Box 26732, Tucson, AZ 85726

MatTHEW MalKan: Institute of Astronomy, University of Cambridge, Cambridge, England CB3 0HA 